https://helda.helsinki.fi

\title{
Taurodontism in the first permanent molars in Van der Woude syndrome compared to isolated cleft palate
}

Awadh, Wael

2021-02

Awadh , W , Pegelow , M , Heliövaara , A \& Rice , D P 2021, ' Taurodontism in the first permanent molars in Van der Woude syndrome compared to isolated cleft palate ' , pÿEuropean Journal of Orthodontics , vol. 43 , no. 1 , pp. 2935 . https://doi.org/10.1093/ejo/cjaa014

http://hdl.handle.net/10138/339711

https://doi.org/10.1093/ejo/cjaa014

acceptedVersion

Downloaded from Helda, University of Helsinki institutional repository.

This is an electronic reprint of the original article.

This reprint may differ from the original in pagination and typographic detail.

Please cite the original version. 


\title{
Taurodontism in the first permanent molars in Van der Woude syndrome compared to isolated cleft palate
}

\author{
Wael Awadh 1,2, Marie Pegelow ${ }^{3}$, Arja Heliövaara ${ }^{4}$, and David P. Rice ${ }^{1, \odot}$
}

'Orthodontics, Department of Oral and Maxillofacial Diseases, University of Helsinki and Helsinki University Hospital, Helsinki, Finland 'Division of Orthodontics, Department of Preventive Dental Sciences, College of Dentistry, Jazan University, Jazan, Saudi Arabia ${ }^{3}$ Division of Orthodontics, Department of Dental Medicine, Karolinska Institutet, Stockholm, Sweden ${ }^{4}$ Cleft Palate and Craniofacial Center, Department of Plastic Surgery, Helsinki University Hospital, Helsinki, Finland

Correspondence to: David P. Rice, Orthodontics, Department of Oral and Maxillofacial Diseases, Biomedicum 1, PL 63 (Haartmaninkatu 8), 00014 University of Helsinki, Finland. E-mail: David.Rice@Helsinki.fi

\section{Summary}

Objectives: To analyse prevalence, pattern, and severity of taurodontism in individuals with Van der Woude syndrome (VWS) exhibiting cleft palate and compare with aged-matched nonsyndromic cleft palate (NSCP) and non-cleft controls.

Materials and methods: One hundred and seventy-eight dental panoramic tomographs (DPTs) (105 girls and 73 boys) consisting of 42 VWS patients $(\bar{x}=8.55 \pm 1.02$ years), 42 NSCP patients $(\bar{x}=8.59 \pm 1.02$ years), and 94 normative non-cleft children $(\bar{x}=8.79 \pm 1.16$ years $)$ were assessed and their first permanent molars evaluated. Measurement 3 of the taurodontism index developed by Shifman and Chanannel with the Tulensalo modification was used. Prevalence, pattern, and severity were compared between groups. Statistical differences were determined by one-way analysis of variance and Fisher test. Repeatability was calculated by Cohens Kappa test.

Results: The prevalence of taurodontic molars was $59.5 \%$ in VWS, $45.2 \%$ in NSCP, and $26.6 \%$ in non-cleft controls. The prevalence and severity of taurodontism in VWS and NSCP were significantly higher than in non-cleft children in all first permanent molars. There was no significant difference in prevalence and severity between VWS and NSCP. The odds for having taurodontism in the VWS group was approximately double compared to the NSCP group. Most of the taurodontic molars showed hypotaurodontism and taurodontism occurred bilaterally more frequently than unilaterally. Conclusion: This study shows a higher prevalence of taurodontism in VWS and NSCP. Most taurodontic molars are hypotaurodontic and most occur bilaterally.

\section{Introduction}

Taurodontism (Online Mendelian Inheritance in Man OMIM $\# 272700$ ) is a condition in which the pulp chambers of multi-rooted teeth are elongated occluso-apically and characterized by apical displacement of the root bi- or trifurcation (1). This is accompanied by a reduced or absent constriction at the cemento-enamel junction (2). Taurodontism is seen in both permanent and primary teeth, although less commonly in the latter $(2,3)$. In the most extreme form, a molar tooth may, instead of having several roots, have only one root with an enlarged pulp chamber (4). Crown to root ratio was used to determine

Editors' comment: This manuscript went through a double blinded submission and review process. This meant that the reviewers were blinded from the authors' identity and the authors were blinded from the submission process including the reviewers' identity. As a co-author of this publication this blinding also applied to David Rice Editor of the EJO. 
the taurodontism severity index, and the taurodontism divided into subgroups according to the index of severity (5). He introduced the terms hypotaurodont, mesotaurodont, and hypertaurodont (5). Taurodontic teeth are also classified as pyramidal, conical and fused in their morphology (4). The prevalence of taurodontism varies widely in normal populations from $0.3 \%$ in Sweden to $2-3 \%$ in Indian, Eskimos, and Native Americans to $46 \%$ in Chinese population $(6,7)$. The frequency of hypo-, meso-, and hypertaurodontism is $2.8 \%, 0.4 \%$, and $0.57 \%$, respectively, in Caucasian Americans (8). Inheritance as a dominant trail has been suggested (9). Taurodontism has been reported in both the primary and the permanent dentation, and in premolar as well as molar teeth (1). It can affect single or multiple teeth and occur either unilaterally or bilaterally (1). Maxillary second molars followed by mandibular second molars are the most commonly teeth effected, with maxillary third molars being the least affected in an Indian population (6). The diagnoses of taurodontism can be important when treating an individual for caries, particularly if endodontic therapy or extraction are being considered (10). Taurodontism may also complicate prosthetic and orthodontic treatment (10).

An increased prevalence of taurodontism in non-syndromic cleft lip and/or palate (NSCL/P) has been reported $(11,12)$. Taurodontism has been recorded equally in twins discordant and concordant for clefts, $41 \%$ (11). In a relatively large sample (724) of non-syndromic cleft patients, they found taurodontism in $60.4 \%$ of cleft lip and palate patients, $62.4 \%$ of cleft lip only patients, and $67 \%$ in patients with cleft palate only, compared to $42.8 \%$ of a control population (13). In general individuals with cleft palate only seem to have a higher frequency of taurodontism than individuals with either cleft lip and palate or cleft lip only $(11,13)$.

Taurodontism is associated with several other craniofacial developmental conditions including Amelogenesis imperfecta (Types IA and IV) (OMIM \#104530 and \#104510) and Trichodentoosseous syndrome (OMIM \#190320) (14). Taurodontism is also seen in individuals with X linked hypohidrotic ectodermal dysplasia (OMIM \#305100). These individuals are characterized by sparse hair, sweaty anomalies as well as hypodontia (15). Other conditions with taurodontism as an associated feature include Down syndrome (OMIM \#190685), Williams-Beuren syndrome (OMIM \#194050), Klinefelter syndrome and 47,XXX karyotype (16-18).

Van der Woude syndrome (VWS) (OMIM \#119300) is an autosomal dominant disorder affecting 1 in 35 000-100 000 people (19). VWS is the most common syndromic form of orofacial clefting accounting for $1-2 \%$ of all cleft cases (20). In addition to clefting, individuals with VWS commonly exhibit lower lip pits and or lip sinuses and hypodontia (21). CL/P is found in 50\% and hypodontia in $25 \%$ of patients with VWS. Regarding hypodontia, the most frequent pattern of missing teeth are upper second premolars, lower second premolars, and upper lateral incisors (22). Mutations in IRF6 and GRHL3 have been shown to be the major genetic causes of VWS $(23,24)$.

Taurodontism has previously been reported in a cohort of 13 VWS patients, in which 6 exhibited taurodontism (25). The aim of this study was to analyse and compare the prevalence pattern and severity of taurodontic molars in a large cohort patients born with VWS, with aged-matched patients born with non-syndromic cleft palate (NSCP) and age-matched non-cleft controls.

\section{Materials and methods}

\section{Study design, setting, and participants}

This retrospective cross-sectional cohort study was performed on dental panoramic tomographs (DPTs) of 378 children consisting of
52 DPTs of patients born with VWS exhibiting cleft palate (taken 1971-2013), 52 DPTs of age-matched patients born with NSCP (isolated cleft palate) (taken 1990-2013), and 274 DPTs of noncleft aged-matched children (taken 1965-8). The cleft palate type in both VWS and NSCP groups had equally subdivided types $(70.5 \%$ hard palate cleft, $13.7 \%$ soft palate cleft, and $15.6 \%$ submucous cleft palate). Patients were diagnosed and treated at the Cleft Palate and Craniofacial Centre, Department of Plastic Surgery, Helsinki University Hospital and the research conducted at the Cleft Palate and Craniofacial Centre, Faculty of Medicine, University of Helsinki, Finland. For all the children with VWS a geneticist or an experienced specialist verified the diagnosis. DPTs of the NSCP group were randomly selected from patients aged 8 years from the archive of the cleft centre using the free-random selection program 'Pick Me!' (By Donation Coder forum, 2009). The examiners were blinded from the group identity when analysing the DPTs. DPTs of the non-cleft group were from the normative Finnish database. This collection consists of DPTs taken of 1017 children between 2 and 19 years of age. The material was collected from school children in Helsinki, Finland (26). To reduce the risk of selection bias it was decided to use the whole 6-10 years old cohort from the normative Finnish collection.

The inclusion criteria were: All subjects in the syndrome group had VWS only. DPT was taken between the age of 6 and 10 years. DPT radiograph for diagnosis was of good quality. The exclusion criteria were: less than half of the roots were developed. One or more first permanent molars had been extracted or had a large restoration. Poor quality of radiographs. If the patient was diagnosed with another cleft type in addition to the cleft palate, submucous cleft; and if the patient had an additional syndrome combined with VWS.

After following the study criteria, 10 radiographs were excluded from the VWS sample, 10 from the NSCP sample, and 180 from the non-cleft sample. The reasons for exclusion were the poor quality of the radiograph, restored, decayed, or missing first molar teeth. This resulted in 42 radiographs in the VWS group for evaluation, 42 radiographs in the NSCP group, and 94 radiographs in the normative Finnish control group. For all groups the mean age and range were similar (Table 1). The Federation Dentaire Internationale (FDI) tooth identifcation system was used.

\section{Ethical issues}

Helsinki and Uusimaa Hospital District approved study protocol HUS/358/2018. The principles of the declaration of Helsinki were followed.

\section{Measurements of dental features by DPT}

Maxillary and mandibular first permanent molars were evaluated for taurodontism, assessing both the prevalence pattern and severity. Tooth outlines were drawn from radiographs using tracing paper and a pencil $(0.5 \mathrm{~mm})$. We evaluated the prevalence and severity of taurodontic molars by using 'measurement 3', the distance between the connecting line of the cemento-enamel junctions and the highest point of the floor of the pulp chamber from the taurodontism index described by Shifman and Chanannel (29) and modified by Tulensalo et al. (30). If the measurement 3 of the tooth was lower than $3.5 \mathrm{~mm}$, the tooth was classified as a non-taurodontic molar. Taurodontic teeth were classified in to three subcategories. Hypotaurodontism was defined as a range of 3.5-5.0 mm, mesotaurodontism 5.5-7.0 mm, and hypertaurodontism $7.5 \mathrm{~mm}$ or over (30). Measurements were recorded to the 
nearest $0.5 \mathrm{~mm}$. Age, gender, and group were blinded during the examination.

\section{Repeatability study}

Forty five DPTs (15 DPTs from each group) were randomly selected using the free-random selection program 'Pick Me!' (By Donation Coder forum, 2009). The same investigator (WA) traced the radiographs twice with a 48 hours interval. Repeatability was calculated using Cohens Kappa test.

\section{Statistics}

Statistical analysis was performed using SPSS (version 22.0, SPSS Inc., Chicago, Illinois, USA). One-way analysis of variance was used to test the severity of taurodontism, to analyse differences between the groups. Fisher test was used to check the prevalence of taurodontism. Univariable logistic regression was used to analyse the interaction of variables 'group', 'age', and 'gender' effects for presence of taurodontism. A $P$-value $<0.05$ was considered to indicate a significant difference. The statistical analyses were performed by a statistician.

\section{Results}

\section{Patterns of taurodontism}

The prevalence of taurodontic molars was ( 25 out of $42,59.5 \%$ ) in individuals with VWS, (19 out of $42,45.2 \%$ ) in individuals with NSCP, and $(25$ out of $94,26.6 \%)$ in non-cleft controls. Younger children (mean age $8.47 \pm 0.97$ years) had significantly more taurodontism than older (mean age $8.82 \pm 1.16$ years) $(P=0.042)$. Taurodontism was found more frequently bilaterally $(85.5 \%)$ than unilaterally $(14.5 \%)$. Taurodontism was observed most commonly in all four first permanent molars (33\%), thereafter both upper first molars $(22 \%)$, and then both lower first molars $(15 \%)$ (Table 2).

\section{Prevalence of taurodontism by tooth and groups}

The prevalence of taurodontism can either be calculated as the frequency of individuals with taurodontic teeth in the study population or as the ratio of taurodontic teeth to the total number of examined teeth $(6,12,13,31)$. In this study, we calculated the prevalence of taurodontism as the frequency of patients who had taurodontism in each first permanent molar. We did not evaluate the second

Table 1. Description of the cohort and subject groups.

\begin{tabular}{lllllr}
\hline Group & $n(178)$ & Gender & $n$ & Age range (years) & $\bar{x}$ \\
\hline VWS & 42 & M & 13 & $6.65-10.81$ & 8.55 \\
NSCP & 42 & F & 29 & & 1.02 \\
\multirow{2}{*}{ Non-cleft control } & 94 & M & 14 & $6.24-10.83$ & 8.59 \\
& & F & 28 & $6.35-10.67$ & 8.79 \\
\end{tabular}

$n$, sample size; $\bar{x}$, mean in years; $\mathrm{M}$, male; $\mathrm{F}$, female; SD, standard deviation.

Table 2. Prevalence and pattern of taurodontism in VWS and NSCP.

\begin{tabular}{|c|c|c|c|c|}
\hline Category & No taurodontism & Taurodontism & Total & $P$-value \\
\hline$n$ (all samples) & 109 & 69 & 178 & \\
\hline \multicolumn{5}{|l|}{ Groups, $n(\%)$} \\
\hline Non-cleft & $69(73.4)$ & $25(26.6)$ & $94(100.0)$ & \\
\hline VWS & $17(40.4)$ & $25(59.5)$ & $42(100.0)$ & \\
\hline NSCP & $23(54.7)$ & $19(45.2)$ & $42(100.0)$ & \\
\hline Chronological age, mean (SD) & $8.82(1.16)$ & $8.47(0.97)$ & $8.68(1.10)$ & 0.042 \\
\hline Mean taurodontism (measurement $3, \mathrm{~mm}$ ) (SD) & $2.44(0.38)$ & $3.61(0.55)$ & $2.90(0.73)$ & $<0.001$ \\
\hline \multicolumn{5}{|l|}{ Pattern of taurodontism for all subjects, $n(\%)$} \\
\hline FDI tooth notation & $109(100.0)$ & $0(0.0)$ & $109(61.2)$ & \\
\hline 46 & $0(0.0)$ & $3(4.3)$ & $3(1.7)$ & \\
\hline 36 & $0(0.0)$ & $1(1.4)$ & $1(0.6)$ & \\
\hline 36,46 & $0(0.0)$ & $10(14.5)$ & $10(5.6)$ & \\
\hline 26 & $0(0.0)$ & $5(7.2)$ & $5(2.8)$ & \\
\hline 26,36 & $0(0.0)$ & $2(2.9)$ & $2(1.1)$ & \\
\hline $26,36,46$ & $0(0.0)$ & $1(1.4)$ & $1(0.6)$ & \\
\hline 16 & $0(0.0)$ & $1(1.4)$ & $1(0.6)$ & \\
\hline 16,36 & $0(0.0)$ & $1(1.4)$ & $1(0.6)$ & \\
\hline $16,36,46$ & $0(0.0)$ & $1(1.4)$ & $1(0.6)$ & \\
\hline 16,26 & $0(0.0)$ & $15(21.7)$ & $15(8.4)$ & \\
\hline $16,26,46$ & $0(0.0)$ & $1(1.4)$ & $1(0.6)$ & \\
\hline $16,26,36$ & $0(0.0)$ & $5(7.2)$ & $5(2.8)$ & \\
\hline $16,26,36,46$ & $0(0.0)$ & $23(33.3)$ & $23(12.9)$ & \\
\hline
\end{tabular}

Taurodontism is more common in both VWS and NSCP compared to non-cleft controls. The most common pattern of taurodontism is that all maxillary and mandibular first permanent molars are affected followed by either both maxillary first permanent molars or both mandibular first permanent molars. $n$, sample size; SD, standard deviation. 
permanent molars, because in our patients the second permanent molar development was still immature.

Taurodontism was significantly more prevalent in individuals with either VWS ( 25 out of $42,59.5 \%$ ), or NSCP (19 out of $42,45.2 \%$ ) compared to non-cleft controls (25 out of $94,26.6 \%$ ) (Figure 1). When considering each molar, the prevalence of taurodontism in both the VWS and NSCP groups was significantly higher than in the non-cleft group for all first maxillary and mandibular permanent molars, $(16, P \leq 0.005),(26, P=0.050),(36, P \leq 0.001)$, and $(46, P \leq 0.001)$ (Table 3 and Figure 2$)$.

The highest prevalence of taurodontism in VWS was sequentially found in mandibular left first permanent molars $(50 \%)$, then maxillary first permanent molars $(42.9 \%)$, then mandibular right first permanent molars $(40.5 \%$ ) (Table 3 and Figure 2). The highest prevalence of taurodontism in NSCP was found in mandibular left first permanent molars $(38.1 \%)$, then maxillary first permanent molars and mandibular right first permanent molar in same percentage $(31 \%)$ (Table 3 and Figure 2). The prevalence of taurodontism in the NSCP group was not significantly different compared to VWS (Supplementary Table 1).The highest prevalence of taurodontism in non-cleft children was sequentially found in maxillary left first permanent molars $(22.3 \%)$, then maxillary right first permanent molars $(17 \%)$, mandibular right first permanent molars $(9.6 \%)$, and mandibular left first permanent molars (7.4\%) (Table 3 and Figure 2).

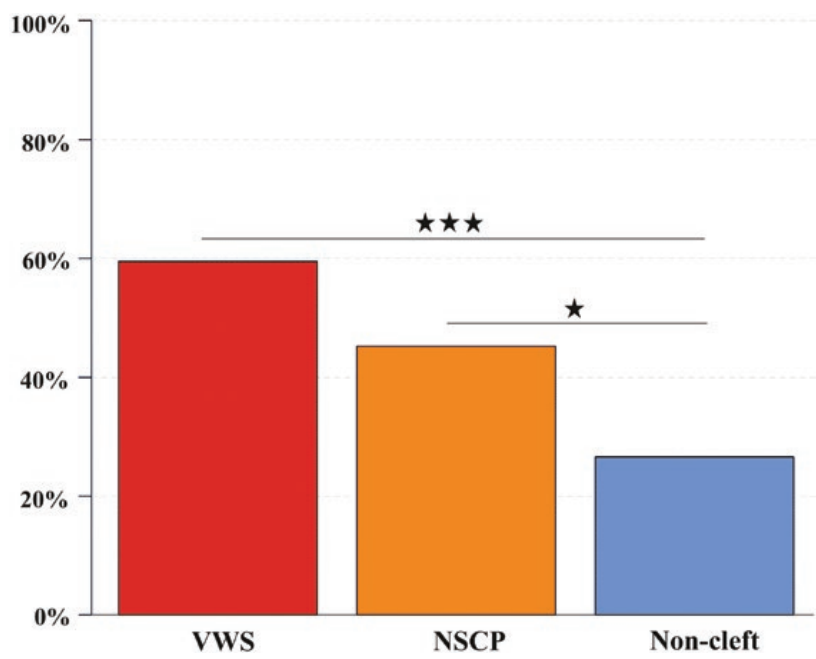

Figure 1. Taurodontism is significantly more prevalent in individuals with either VWS or NSCP compared to non-cleft controls. ${ }^{*} P<0.05$, $* * * P<0.001$.
Effects variable to presence taurodontism using univariable logistic regression

Both VWS and NSCP groups had significantly higher odds ratio for taurodontism compared to the non-cleft group. The VWS group had 4.06 times higher odds for having taurodontism compared to the non-cleft group $(P=0.000)$. The NSCP group had 2.28 times higher odds of having taurodontism compared to the non-cleft group $(P=0.030)$. There was no significant difference between genders. However, males had slight higher odds ratio than females (not significant) (Supplementary Table 1).

\section{The severity of taurodontism by tooth and groups}

Both VWS and NSCP groups had more severe taurodontism compared to the non-cleft as a reference control group in all first maxillary and mandibular permanent molars, $(16, P \leq 0.001),(26$, $P=0.024),(36, P \leq 0.001)$, and $(46, P \leq 0.001)$ (Table 4). However, there was no difference in severity between VWS and NSCP groups for each of the first permanent molars (Supplementary Table 2). Four of the taurodontic molars from the groups were mesotaurodontic, all the other were hypotaurodontic (Table 4).

\section{Repeatability study}

Inter-observer repeatability by Cohens Kappa test between two measurements showed excellent agreement for all molars. The range of Kappa values was 0.81-0.93.

\section{Discussion}

\section{Taurodontism in VWS and NSCP}

Our data demonstrate a high prevalence of taurodontism in both the VWS and NSCP cohorts. Taurodontism has been reported in VWS previously (25). They documented 6 subjects with at least 1 taurodontic tooth in a cohort of 13 VWS patients $(46 \%)$. In our larger sample, the prevalence of taurodontism was $59.5 \%$. We also documented the pattern of taurodontism in the first permanent molars and found taurodontism to be more commonly bilateral than unilateral. Most of the taurodontic molars were hypotaurodontic in form.

We found that $45.2 \%$ of our NSCP cohort exhibited taurodontism. In a cohort of 224 adult patients with NSCP, they found a taurodontic frequency of $67.0 \%$ (13). They also found taurodontism in $60.4 \%$ of subjects with unilateral cleft lip and palate, and $62.4 \%$ in unilateral cleft lip patients, and $42.8 \%$ in a non-cleft control group. In the whole cleft population they found that the upper second permanent molar teeth were most often affected (13). In a case-control study of 88 subjects with NSCL/P, 36 patients (40.9\%) exhibited

Table 3. Comparisons of the prevalence of taurodontism between groups in each studied tooth.

\begin{tabular}{|c|c|c|c|c|c|}
\hline Tooth FDI notation & $\begin{array}{l}\text { Non-cleft (Ref.) } \\
(n=94) \\
n(\%)\end{array}$ & $\begin{array}{l}\text { VWS } \\
\begin{array}{l}(n=42) \\
n(\%)\end{array}\end{array}$ & $\begin{array}{l}\text { NSCP } \\
\begin{array}{l}(n=42) \\
n(\%)\end{array}\end{array}$ & $P$-value & Test \\
\hline 16 & $16(17.0)$ & 18 (42.9) & $13(31.0)$ & $0.005^{* *}$ & Fishers \\
\hline 26 & $21(22.3)$ & $18(42.9)$ & $13(31.0)$ & $0.050^{*}$ & Fishers \\
\hline 36 & $7(7.4)$ & $21(50.0)$ & $16(38.1)$ & $<0.001 * * *$ & Fishers \\
\hline 46 & $9(9.6)$ & $17(40.5)$ & $13(31.0)$ & $<0.001 * * *$ & Fishers \\
\hline
\end{tabular}

A higher prevalence of taurodontism in both VWS and NSCP compared to non-cleft controls for every tooth assayed (16, 26, 36, and 46). $n$, sample size.

$* P<0.05$.

$* * P<0.01$.

$* * P<0.001$ 
taurodontism compared to $23.3 \%$ of control group (12). Most teeth were classified as hypotaurodontic, followed by mesotaurodontic then hypertaurodontic in form (12). These wide variations in taurodontic frequency may reflect the populations sampled, and the method used to determine taurodontism. However, they also may be a reflection of the teeth evaluated and of the age patients sampled. For both non-cleft and all cleft types, taurodontism is more frequent in second molars than in first molars (32). So as the second molars develop after the first, the older the patient, the more likely they are to have a taurodontic molar. In our study, we only investigated first molars as at the age of the participants, 8 years, as the second molar is still at an early stage of development.

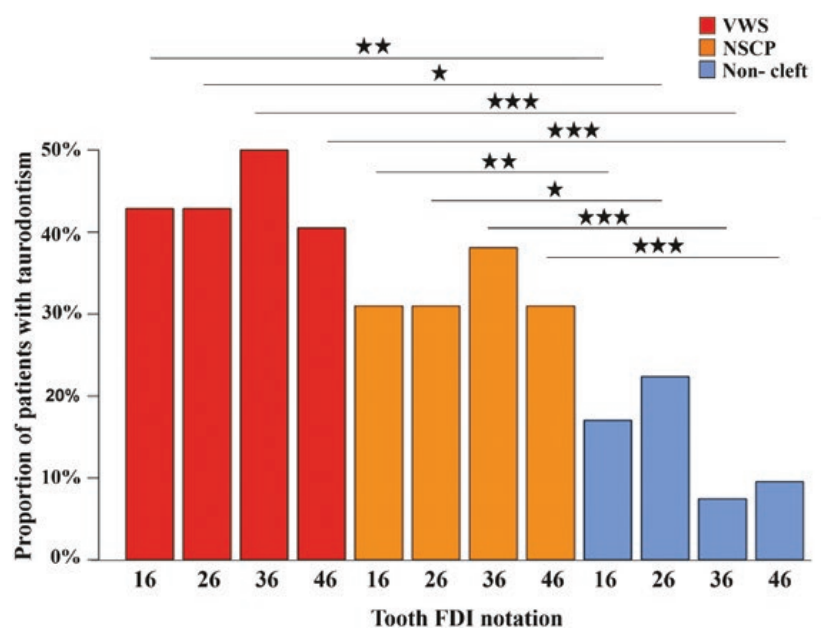

Figure 2. Comparison of the prevalence of taurodontism between groups for each tooth. For every tooth assayed $(16,26,36$, and 46) there is a higher prevalence of taurodontism in both VWS and NSCP compared to non-cleft controls. ${ }^{*} P<0.05,{ }^{*} P<0.01,{ }^{*} * * P 0.001$.
In our study material symmetric bilateral taurodontism was more common than unilateral. Most other studies have found a similar correlation, that most taurodontism cases are bilateral (29, 32 ). However, in other study they found that nearly half of their cases were unilateral (33). Furthermore, we found that most of the cases of taurodontism were hypotaurodontic in morphology. And this also correlates with previous findings (34).

There is conflicting literature on the influence of gender of taurodontism. Some studies have found a significantly higher prevalence in females $(4,35)$. However our study and many other studies contrast this, with no significant difference noted between genders (31, $36,37)$. It should be noted however, that taurodontism has been associated with $\mathrm{X}$ linked hypohidrotic ectodermal dysplasia and the additional X chromosome in 47,XXY and 47,XXX karyotypes (15, 17, 18).

\section{The method of assessing taurodontism}

In this study, we used a method originally described by Blumberg et al. and adapted by Shifman and Chanannel $(27,29)$ and Tulensalo et al. (30). The Tulensalo modification compensated for the different magnification of DPT radiographs compared to intraoral radiographs. This method has been shown to be reliable in assessing taurodontism in the developing dentition (30). Many other methods for assessing taurodontism have been developed $(6,35,38-40)$. Notably measuring the ratio of the crown height to the total tooth length. However, for this method to be used, the root needs to be fully developed and this was therefore not applicable to our sample, aged 8 years $(33,41)$.

\section{The aetiology of taurodontism in VWS}

The features of VWS include both orofacial clefting and hypodontia. Both these phenotypes are independently associated with taurodontism (33). Moreover, taurodontism has been described in patients with incisor-premolar hypodontia and oligodontia $(42,43)$.

Table 4. Comparison of the severity of taurodontism between groups.

\begin{tabular}{|c|c|c|c|c|c|c|c|c|}
\hline $\begin{array}{l}\text { FDI tooth } \\
\text { notation }\end{array}$ & & $\begin{array}{l}\text { Severity of } \\
\text { taurodontism }\end{array}$ & $\begin{array}{l}\text { Non-cleft } \\
(n=94) \\
\text { (Ref.) }\end{array}$ & $\begin{array}{l}\text { VWS } \\
(n=42)\end{array}$ & $\begin{array}{l}\text { NSCP } \\
(n=42)\end{array}$ & $\begin{array}{l}\text { Total } \\
(n=178)\end{array}$ & $P$-value & Test \\
\hline \multirow[t]{4}{*}{16} & $\begin{array}{l}\text { Mean (SD) } \\
\text { mm }\end{array}$ & & $2.63(0.72)$ & $3.17(0.76)$ & $2.98(0.85)$ & & $<0.001 * * *$ & $t$-test \\
\hline & $n(\%)$ & Нуро & $16(17)$ & $18(42.9)$ & $13(31)$ & $47(26.4)$ & & \\
\hline & & Meso & - & - & - & - & & \\
\hline & & Hyper & - & - & - & - & & \\
\hline \multirow[t]{4}{*}{26} & $\begin{array}{l}\text { Mean (SD) } \\
\text { mm }\end{array}$ & & $2.74(0.71)$ & $3.11(0.84)$ & $3.02(0.93)$ & & $0.024 *$ & $t$-test \\
\hline & $n(\%)$ & Нуро & $21(22.3)$ & $18(42.9)$ & $13(31.0)$ & $52(29.2)$ & & \\
\hline & & Meso & - & - & - & - & & \\
\hline & & Hyper & - & - & - & - & & \\
\hline \multirow[t]{4}{*}{36} & $\begin{array}{l}\text { Mean (SD) } \\
\text { mm }\end{array}$ & & $2.59(0.52)$ & $3.32(0.97)$ & $3.31(1.02)$ & & $<0.001^{* * *}$ & $t$-test \\
\hline & $n(\%)$ & Нуро & $7(7.4)$ & $21(50.0)$ & 15 (15.9) & $43(24.1)$ & & \\
\hline & & Meso & - & - & $1(1)$ & $1(0.5)$ & & \\
\hline & & Hyper & - & - & - & - & & \\
\hline \multirow[t]{4}{*}{46} & $\begin{array}{l}\text { Mean (SD) } \\
\text { mm }\end{array}$ & & $2.63(0.57)$ & $3.29(0.92)$ & $3.24(0.96)$ & & $<0.001^{* * *}$ & $t$-test \\
\hline & $n(\%)$ & Нуро & $9(9.6)$ & $16(38)$ & $11(11.7)$ & $36(20.2)$ & & \\
\hline & & Meso & - & $1(2.3)$ & $2(2.1)$ & $3(1.6)$ & & \\
\hline & & Hyper & - & - & - & - & & \\
\hline
\end{tabular}

All taurodontic teeth found exhibit hypotaurodontism in all groups, except four mandibular first molars were mesotaurodontism in both VWS and NSCP groups. $* P<0.05$. ${ }^{*} P<0.01$. $* * P<0.001 . n$, sample size; $\mathrm{SD}$, standard deviation. 
Loss of function mutations in IRF6 are important causes of VWS (23). Mutant mice carrying a homolog of the gene mutated in the human VWS which die perinatally, exhibit disrupted palate development and widespread epithelial abnormalities including intraoral adhesions, which are also observed in VWS patients (44). IRF6 is needed for proper periderm development and function. The periderm, the outermost layer of the oral epithelium, has a key role in stopping inappropriate pathological adhesions as well as controlling palatal adhesion and fusion competence (45). To obviate the perinatal death of IRF6 mutant mice and allow the study of the later stages of tooth development, mice carrying mutant IRF6 under that control of a different promoter (Pitx2Cre) have been generated (46). These mice have been reported to have dental morphological defects including severe taurodontism, as well as peg-shaped teeth, C-shaped molars and loss of separate mesial and distal first molar canals with transverse mid-root region of the mandibular molar. In addition, Pitx2Cre/+; Irf6fl/+ mice also exhibit hypodontia, supplemental incisors. IRF6 is expressed in the developing oral/palatal epithelium, the dental epithelium and in the ameloblasts, and clearly has a functional role during palate and tooth morphogenesis and dental hard tissue formation and can go some way to explaining the multiple craniofacial phenotypes seen in patients with VWS $(28,46,47)$.

\section{Strengths and limitations}

The main strength of this research is the large cohort of the rare condition, VWS. The data with standardized records were collected over a 42-year period. Also of merit are appropriate control groups, which are taken from the same general population and geographical region as the test sample and are well matched for age and gender.

One limitation concerning the non-cleft control sample was the large 'drop out' rate of the radiographs. Another limitation was that while there was overlap in the historical time periods over which the radiographs were taken, there were still differences in the time periods between the groups.

\section{Conclusion}

The prevalence and severity of taurodontism in VWS and NSCP were significantly higher than in non-cleft children of all first permanent molars. Most of the taurodontic molars showed hypotaurodontism and taurodontism occurred more frequently bilateral than unilateral.

\section{Supplementary material}

Supplementary data are available at European Journal of Orthodontics online.

Supplementary Table 1 . The presence of taurodontism in VWS and NSCP compared to non-cleft individuals using univariable logistic regression. There is an increased odds ratio for both VWS and NSCP compared to the non-cleft group. There is no statistical difference in the odds ratio between genders. $n$, sample size.

Supplementary Table 2. Comparison of severity of taurodontism between VWS and NSCP groups. There is no statistical difference in severity of taurodontism between VWS and NSCP groups for every tooth assayed $(16,26,36$, and 46$)$. $* P<0.05, * * P<0.01, * * P$ $<0.001$.

\section{Acknowledgements}

We are greatly thankful to E. Hagel for her statistical assistance.

\section{Funding}

College of Dentistry at Jazan University in Saudi Arabia for their support funding (WA), and the Helsinki University Hospital Research Foundation for funding (DR).

\section{Conflicts of interest}

The authors have no conflict of interest.

\section{References}

1. Haskova, J.E., Gill, D.S., Figueiredo, J.A., Tredwin, C.J. and Naini, F.B. (2009) Taurodontism—a review. Dental Update, 36, 235-236, 239.

2. Luder, H.U. (2015) Malformations of the tooth root in humans. Frontiers in Physiology, 6, 307.

3. Keith, A. (1913) Problems relating to the teeth of the earlier forms of prehistoric man. Proceedings of the Royal Society of Medicine, 6, 103-124.

4. Sarr, M., Toure, B., Kane, A.W., Fall, F. and Wone, M.M. (2000) [Taurodontism and the pyramidal tooth at the level of the molar. Prevalence in the Senegalese population 15 to 19 years of age]. Odonto-Stomatologie Tropicale $=$ Tropical Dental Journal, 23, 31-34.

5. Shaw, J.C. (1928) Taurodont teeth in South African races. Journal of Anatomy, 62, 476-498.1.

6. Gupta, S.K. and Saxena, P. (2013) Prevalence of taurodontism and its association with various oral conditions in an Indian population. Oral Health \& Preventive Dentistry, 11, 155-160.

7. MacDonald-Jankowski, D.S. and Li, T.T. (1993) Taurodontism in a young adult Chinese population. Dento Maxillo Facial Radiology, 22, 140-144.

8. Sood, P.B. and Sood, M. (1992) Taurodontism and pyramidal molars. Journal of the Indian Society of Pedodontics and Preventive Dentistry, 10, $25-27$.

9. Goldstein, E. and Gottlieb, M.A. (1973) Taurodontism: familial tendencies demonstrated in eleven of fourteen case reports. Oral Surgery, Oral Medicine, and Oral Pathology, 36, 131-144.

10. Dineshshankar, J., Sivakumar, M., Balasubramanium, A.M., Kesavan, G., Karthikeyan, M. and Prasad, V.S. (2014) Taurodontism. Journal of Pharmacy \& Bioallied Sciences, 6, S13-S15.

11. Laatikainen, T. and Ranta, R. (1996) Occurrence of the Carabelli trait in twins discordant or concordant for cleft lip and/or palate. Acta Odontologica Scandinavica, 54, 365-368.

12. Melo Filho, M.R., Nogueira dos Santos, L.A., Barbosa Martelli, D.R., Silveira, M.F., Esteves da Silva, M., de Barros, L.M., Coletta, R.D. and Martelli-Júnior, H. (2015) Taurodontism in patients with nonsyndromic cleft lip and palate in a Brazilian population: a case control evaluation with panoramic radiographs. Oral Surgery, Oral Medicine, Oral Pathology and Oral Radiology, 120, 744-750.

13. Weckwerth, G.M., Santos, C.F., Brozoski, D.T., Centurion, B.S., Pagin, O., Lauris, J.R., Carvalho, I.M. and Neves, L.T. (2016) Taurodontism, root dilaceration, and tooth transposition: a radiographic study of a population with nonsyndromic cleft lip and/or palate. The Cleft Palate-Craniofacial Journal, 53, 404-412.

14. Crawford, P.J., Evans, R.D. and Aldred, M.J. (1988) Amelogenesis imperfecta: autosomal dominant hypomaturation-hypoplasia type with taurodontism. British Dental Journal, 164, 71-73.

15. Crawford, P.J., Aldred, M.J. and Clarke, A. (1991) Clinical and radiographic dental findings in $\mathrm{X}$ linked hypohidrotic ectodermal dysplasia. Journal of Medical Genetics, 28, 181-185.

16. Axelsson, S. (2005) Variability of the cranial and dental phenotype in Williams syndrome. Swedish Dental Journal, (Suppl), 3-67.

17. Varrela, J. and Alvesalo, L. (1988) Taurodontism in 47,XXY males: an effect of the extra X chromosome on root development. Journal of Dental Research, 67, 501-502.

18. Varrela, J. and Alvesalo, L. (1989) Taurodontism in females with extra $\mathrm{X}$ chromosomes. Journal of Craniofacial Genetics and Developmental Biology, 9, 129-133. 
19. Gorlin, R.J., Cohen Jr, M.M. and Hennekam, R.C. (2001) Syndromes of the Head and Neck. Oxford University Press, New York, 4th edn.

20. Burdick, A.B., Bixler, D. and Puckett, C.L. (1985) Genetic analysis in families with van der Woude syndrome. Journal of Craniofacial Genetics and Developmental Biology, 5, 181-208.

21. Van der Woude, A. (1954) Fistula labii inferioris congenita and its association with cleft lip and palate. American Journal of Human Genetics, 6, 244-256.

22. Rizos, M. and Spyropoulos, M.N. (2004) Van der Woude syndrome: a review. Cardinal signs, epidemiology, associated features, differential diagnosis, expressivity, genetic counselling and treatment. European Journal of Orthodontics, 26, 17-24.

23. Kondo, S., et al. (2002) Mutations in IRF6 cause Van der Woude and popliteal pterygium syndromes. Nature Genetics, 32, 285-289.

24. Peyrard-Janvid, M., et al. (2014) Dominant mutations in GRHL3 cause Van der Woude Syndrome and disrupt oral periderm development. American Journal of Human Genetics, 94, 23-32.

25. Nawa, H., Oberoi, S. and Vargervik, K. (2008) Taurodontism and Van der Woude syndrome. Is there an association? The Angle Orthodontist, 78, 832-837.

26. Haavikko, K. and Helle, A. (1974) A cephalometric study of facial types and occlusion classes and relation between them in Finnish children. Proceedings of the Finnish Dental Society. Suomen Hammaslaakariseuran toimituksia, 70, 99-106.

27. Blumberg, J.E., Hylander, W.L. and Goepp, R.A. (1971) Taurodontism: a biometric study. American Journal of Physical Anthropology, 34, 243255.

28. Washbourne, B.J. and Cox, T.C. (2006) Expression profiles of cIRF6, cLHX6 and cLHX7 in the facial primordia suggest specific roles during primary palatogenesis. BMC Developmental Biology, 6, 18.

29. Shifman, A. and Chanannel, I. (1978) Prevalence of taurodontism found in radiographic dental examination of 1,200 young adult Israeli patients. Community Dentistry and Oral Epidemiology, 6, 200-203.

30. Tulensalo, T., Ranta, R. and Kataja, M. (1989) Reliability in estimating taurodontism of permanent molars from orthopantomograms. Community Dentistry and Oral Epidemiology, 17, 258-262.

31. Bürklein, S., Breuer, D. and Schäfer, E. (2011) Prevalence of taurodont and pyramidal molars in a German population. Journal of Endodontics, 37, $158-162$.

32. Laatikainen, T. and Ranta, R. (1996) Taurodontism in twins with cleft lip and/or palate. European Journal of Oral Sciences, 104, 82-86.

33. Seow, W.K. and Lai, P.Y. (1989) Association of taurodontism with hypodontia: a controlled study. Pediatric Dentistry, 11, 214-219.

34. Marques Fernandes, M., Ferreira Silva, R., De Lucena Botelho, T., Ribeiro Tinoco, R.L., Fontanella, V. and Nogueira de Oliveira, R. (2018)
Taurodontism and its forensic value: a case report. The Journal of Forensic Odonto-Stomatology, 36, 40-43.

35. Bronoosh, P., Haghnegahdar, A. and Dehbozorgi, M. (2012) Prevalence of taurodontism in premolars and molars in the South of Iran. Journal of Dental Research, Dental Clinics, Dental Prospects, 6, 21-24.

36. Constant, D.A. and Grine, F.E. (2001) A review of taurodontism with new data on indigenous southern African populations. Archives of Oral Biology, 46, 1021-1029.

37. Jafarzadeh, H., Azarpazhooh, A. and Mayhall, J.T. (2008) Taurodontism: a review of the condition and endodontic treatment challenges. International Endodontic Journal, 41, 375-388.

38. Kan, W.Y., Seow, W.K. and Holcombe, T. (2010) Taurodontism in children with hypodontia and supernumerary teeth: a case control study. Pediatric Dentistry, 32, 134-140.

39. Calvano Küchler, E., De Andrade Risso, P., De Castro Costa, M., Modesto, A. and Vieira, A.R. (2008) Assessing the proposed association between tooth agenesis and taurodontism in 975 paediatric subjects. International Journal of Paediatric Dentistry, 18, 231-234.

40. Witkop, C.J. Jr. (1971) Manifestations of genetic diseases in the human pulp. Oral Surgery, Oral Medicine, and Oral Pathology, 32, 278-316.

41. Gomes, R.R., Habckost, C.D., Junqueira, L.G., Leite, A.F., Figueiredo, P.T., Paula, L.M. and Acevedo, A.C. (2012) Taurodontism in Brazilian patients with tooth agenesis and first and second-degree relatives: a case-control study. Archives of Oral Biology, 57, 1062-1069.

42. Arte, S., Nieminen, P., Apajalahti, S., Haavikko, K., Thesleff, I. and Pirinen, S. (2001) Characteristics of incisor-premolar hypodontia in families. Journal of Dental Research, 80, 1445-1450.

43. Schalk-van der Weide, Y., Steen, W.H. and Bosman, F. (1993) Taurodontism and length of teeth in patients with oligodontia. Journal of Oral Rehabilitation, 20, 401-412.

44. Richardson, R.J., Dixon, J., Malhotra, S., Hardman, M.J., Knowles, L., Boot-Handford, R.P., Shore, P., Whitmarsh, A. and Dixon, M.J. (2006) Irf6 is a key determinant of the keratinocyte proliferation-differentiation switch. Nature Genetics, 38, 1329-1334.

45. Hammond, N.L., Dixon, J. and Dixon, M.J. (2019) Periderm: life-cycle and function during orofacial and epidermal development. Seminars in Cell \& Developmental Biology, 91, 75-83.

46. Chu, E.Y., Tamasas, B., Fong, H., Foster, B.L., LaCourse, M.R., Tran, A.B., Martin, J.F., Schutte, B.C., Somerman, M.J. and Cox, T.C. (2016) Full spectrum of postnatal tooth phenotypes in a novel Irf6 cleft lip model. Journal of Dental Research, 95, 1265-1273.

47. Knight, A.S., Schutte, B.C., Jiang, R. and Dixon, M.J. (2006) Developmental expression analysis of the mouse and chick orthologues of IRF6: the gene mutated in Van der Woude syndrome. Developmental Dynamics: An Official Publication of the American Association of Anatomists, 235, 1441-1447. 